\title{
Symposium summary: stellar populations in bulges
}

\author{
Beatriz Barbuy ${ }^{1}$ \\ ${ }^{1}$ Universidade de São Paulo, IAG, Astronomy Dept., Rua do Matão 1226, São Paulo \\ 05508-900, Brazil \\ email: barbuy@astro.iag.usp.br
}

\begin{abstract}
Stellar populations in the Milky Way and in external galaxies are reviewed, based on the conferences and posters presented at this meeting.
\end{abstract}

Keywords. Galaxy: bulge, Galaxy: stellar content, galaxies: stellar content

\section{Introduction}

Studies of bulges involve essentially three fields of research, dynamics of bars and secular evolution of bars into bulges, $\Lambda$ CDM models on large scale structure and hierarchical clustering forming galaxies, and finally studies of stellar populations, which includes spectroscopy at high resolution in the Milky Way. Central black holes will not be discussed here.

These three communities have shown great progress in studies of bulges, however they do not agree in some important aspects. It is healthy that these studies are not influenced by each other, and eventually a global picture will emerge.

In a comprehensive introductory talk, Freeman (this volume) describes 3 kinds of bulges: a) $r^{1 / 4}$ bulges, that are large and probably result from mergers; b) Milky Way like: near-exponential boxy bulges, likely generated by disk instability processes; c) pseudobulges generated by secular evolution, i.e., dynamical evolution of bars, recalling that it is estimated that $2 / 3$ of disk galaxies have bars (Combes, this volume). On the other hand, the review by Steinmetz (this conference) on simulations of a $\Lambda$ CDM hierarchical clustering universe, showed that bulges are a natural outcome of such calculations, and that there is no problem in forming bulges, on the contrary the problem is not to form bulges. In these models, around $\mathrm{z} \sim 6$ there is rapid infall (within $0.1 \mathrm{Gyr}$ ) and efficient star formation, such that central bulge regions host the oldest stars of a galaxy, and these are relatively metal-rich. Other mechanisms such as minor mergers (Cox, this volume) were also considered.

\section{Galactic bulge}

Baade (1946) detected a large number of "cluster-type" variables in the direction of the cluster NGC 6522 (the so-called "Baade's Window"), and concluded that the stellar populations in that direction were of type II. This led to the concept of a metal-poor bulge for a few decades. Whitford (1978) and Whitford \& Rich (1983) have shown by means of a series of intermediate resolution spectra of bulge $\mathrm{K}$ giants, that instead the bulge is metal-rich.

During the 80's and 90's several papers argued on the bulge being young, whereas others (Frogel 1988 and references therein) suggested it to be old. Based on HST and NTT data, Ortolani et al. (1995) have shown that metal-rich bulge clusters and field are 
nearly coeval with the halo, and such evidences were further demonstrated by Zoccali et al. (2003).

It is clear now that the bulk of the Galactic bulge stellar populations is old and metalrich, and that spectra of old metal-rich stars and integrated spectra of bulge clusters are similar to integrated spectra of elliptical galaxies (e.g. Rich 1988; Bica 1988).

More recently, other important evidences confirm that the Milky Way bulge is old:

a) $[\mathrm{O} / \mathrm{Fe}]$ vs. $[\mathrm{Fe} / \mathrm{H}]$ is overenhanced in bulge field giants, with respect to thick (and thin) disk stars (the latter results are from Bensby et al. 2004), as clearly shown by four groups: Cunha \& Smith (2006) and Rich \& Origlia (2005) used the infrared OH lines, and Zoccali et al. (2006) and Fulbright et al. (2007) used the forbidden [OI]630nm line (see Cunha et al., this volume; Minniti \& Zoccali, this volume). In Fig. 1 by Cunha et al. (this volume) are plotted these four sets of oxygen-to-iron data. It is interesting to note that Kobayashi (this volume) presented a chemical enrichment model based on nucleosynthesis in hypernovae, producing an oxygen enhanced of $[\mathrm{O} / \mathrm{Fe}] \sim 0.3$ for the Galactic bulge.

The same overenhancement is found for $\mathrm{Mg}$ in bulge field giants, with respect to thick and thin disks (McWilliam \& Rich 1994; Minniti \& Zoccali, this volume; Lecureur et al. 2007; Fulbright et al. 2007). Also, bulge planetary nebulae show oxygen overabundances up to 0.2 dex with respect to disk ones (Maciel et al. and references therein, this volume)

b) The maximum velocity over velocity dispersion $\mathrm{V}_{\max } / \sigma$ as a function of bulge (apparent) flattening $\epsilon$ diagram can be used to distinguish between different types of bulges, and in particular to identify pseudobulges that are more rotation dominated than classical bulges (Kormendy, this volume; Kormendy \& Kennicutt 2004). Rich et al. (this volume) presented results for the Galactic bulge pointing to $\mathrm{V}_{\max } / \sigma \approx 0.6$, indicating that it is does not correspond to a pseudo-bulge, but rather to a classical bulge, whereas Minniti \& Zoccali (this volume) finds a somewhat higher value of $\mathrm{V}_{\max } / \sigma \approx 0.65$.

c) Based on 2MASS and MSX satellite data, Mahoney (this volume; see also LópezCorredoira et al. 2007), showed evidences for a clear distinction between bar and bulge. A long flat bar with dimensions $7.8 \mathrm{kpc} \times 1.2 \mathrm{kpc} \times 0.2 \mathrm{kpc}$, and at a position angle of $43^{\circ}$, or $-14^{\circ}<\mathrm{l}<+30^{\circ}, \mathrm{b}<1.5^{\circ}$, is clearly identified. The triaxial bulge is much wider in latitude, observable at $|1|<15^{\circ},|\mathrm{b}|<10^{\circ}$. Still, a dynamical evolution of the bar, transferring gas and stars to the bulge should be occurring, but its effects seem to be still rather limited, as inferred from evidences a) and b) above.

Globular clusters are as well probes of bulge formation. From studies of globular clusters in the local Universe, Forbes (this volume) presented evidences for metal-rich globular clusters to be old, and only a few ones to be formed in merger events. The Sombrero galaxy (Sa-Sb type), for example, has 800 metal-rich globulars, which from their locations, should be a bulge population and not a disk one. He concluded that spheroids/bulges formed early. Let us remind that in the MW about half of the clusters are within the bulge volume, at $4 \mathrm{kpc}$ of the Galactic center. There is still controversy as to whether these clusters being bulge or disk population in the literature. As concerns metal-poor globulars in the bulge, Kim et al. (this conference) presented a study for about 30 of them. We recall that these targets are very interesting, since many of them should be bulge members, and therefore very old, whereas others could be halo.

In summary, Drory's (this volume) overall suggestion that E-S0 galaxies have classical bulges, Sc's pseudo bulges, whereas Sb and Sbc's show both cases seems to be correct.

\section{Nuclear star formation in the Milky Way and other galaxies}

It is well-known that there are young massive stars in the Galactic center, such as the well-known nuclear star clusters Arches, Quintuplet and GC. Cunha et al. (this volume) 
analysed M giants and supergiants within $2 \mathrm{pc}$ of the Galactic center showing that they are $\alpha$-enriched, despite being young massive solar-metallicity stars.

Thomas (this volume) considers that Sa to Sbc galaxies have $[\alpha / \mathrm{Fe}]=0$ to 0.25 , and that these are old bulges like our own Milky Way. But these galaxies show signs of recent star formation induced by disk instabilities, called "rejuvenation". Therefore their integrated light appears younger than the oldest bulges. In fact, according to Yi (this volume), $10 \%$ of E's, $30 \%$ of S0's and $50 \%$ of spirals show residual star formation, occurred in the past 1-2 Gyr. The study by Sil'chenko (this volume), where 80 S0's were studied, confirms that nuclear regions have to be subtracted from the integrated light of their bulges, in order to have a proper indication on the bulk old population.

\section{Other bulges}

Arimoto (this conference) indicated that M31 shows $\alpha$ enhancement, as well as bulges of spirals in general, with $[\alpha / \mathrm{Fe}] \sim 0.2-0.3$ (see also Thomas, this volume; Prugniel et al. 2001). Peletier (this volume) obtained integral field observations of bulges in Sa-Sd's. Lick indices were measured along the surface of each bulge. They obtained $[\alpha / \mathrm{Fe}] \sim 0$ in SbSd's and +0.3 in Sa's; however there are gradients in spirals, both positive or negative, depending on rings and other subcomponents. Given the several components present, Peletier concluded that the situation is confuse.

Koo (this volume) presented the study of a large sample of galaxies at redshifts of $\mathrm{z} \sim 0.8$ obtained within the DEEP project. They found that bulges and E-S0's are very red and likely old at $\mathrm{z} \sim 1 ;<15 \%$ are blue bulges, which are not likely progenirors of the luminous red bulges, since they are fainter. It is to be pointed out that secular evolution models give a poor fit to these observations.

\section{Conclusions}

Advances in observational evidences are on the way to help solving conflicting views on the Milky Way bulge formation. It appears as a classical bulge from stellar populations studies, whereas dynamical simulation modellers predict it to be a pseudo-bulge resulting from disk dynamical instabilities and secular evolution of the bar. MSX observations combined with 2MASS data have defined with precision the dimensions and location of the bar in the Milky Way, making clear that the bulge is much wider in both 1 and $\mathrm{b}$ (Mahoney, this volume). This helps to explain why abundance ratios indicate a classical bulge, since the fields corresponding to Fig. 1 by Cunha et al. (this volume) are located at Baade's Window $\left(\mathrm{l}=1^{\circ}, \mathrm{b}=-4^{\circ}\right)$, NGC 6553 field $\left(\mathrm{l}=5^{\circ}, \mathrm{b}=-3^{\circ}\right)$ and fields along the minor axis at $b=-6^{\circ}$ and $-12^{\circ}$, therefore not in the low latitudes $\left(b<1.5^{\circ}\right)$ of the bar.

Still concerning the Milky Way, a metallicity distribution (Minniti, this volume) spanning from $-1.0<[\mathrm{Fe} / \mathrm{H}]<+0.5$ shows two important aspects: essentially no stars more metal-poor than $[\mathrm{Fe} / \mathrm{H} \approx-1.0$ are found, and 3 metallicity peaks are seen, with the most metal-rich of them around $[\mathrm{Fe} / \mathrm{H}] \approx+0.3$ declining in intensity along the minor axis.

IFU observations of bulges by Peletier (this volume) show that it is possible to distinguish stellar population components in terms of line indices.

Line indices being measured for thousands of galaxies and galaxy bulges in surveys like DEEP (Koo, this volume), 6dF (Colless, this volume), combined to a multi-wavelength effort such as AEGIS (Koo, this volume), should provide important information on the stellar population content and evolution of bulges with redshift. 
In the overall, the combination of expertises present in this meeting, suggests that the interaction between them will eventually lead to a better understanding and global picture of bulges.

\section{Acknowledgements}

Grants from the Brazilian agencies CNPq and FAPESP are acknowledged.

\section{References}

Baade, W. 1946, PASP 58, 249

Bensby, T., Feltzing, S. \& Lundström, I. 2004, A\&A 415, 155

Bica, E. 1988, A\&A 195, 76

Colless, M. 2007, IAU Symp. 245 this volume

Combes, F. 2007, IAU Symp. 245 this volume

Cox, T. J., Younger, J., Hernquist, L., \& Hopkins, P. F. 2007, IAU Symp. 245 this volume

Cunha, K. \& Smith, V. V. 2006, ApJ 651, 491

Cunha, K., Smith, V. V., Sellgren, K., Blum, R. D., Ramírez, S. V., \& Terndrup, D. M. 2007, IAU Symp. 245 this volume

Drory, N. 2007, IAU Symp. 245 this volume

Forbes, D. 2007, IAU Symp. 245 this volume

Freeman, K. 2007, IAU Symp. 245 this volume

Frogel, J. A. 1988, ARA\&A 26, 51

Fulbright, J. P., McWilliam, A., \& Rich, R. M. 2007, ApJ 661, 1152

Kobayashi, C. 2007, IAU Symp. 245 this volume

Koo, D. 2007, IAU Symp. 245 this volume

Kormendy, J. 2007, IAU Symp. 245 this volume

Kormendy, J. \& Kennicutt, R. 2004, ARA\&A 42, 603

Lecureur, A., Hill, V., Zoccali, M., Barbuy, B., Gómez, A., Minniti, D., Ortolani, S., \& Renzini, A. $2007, A \& A 465,799$

López-Corredoira, M., Cabrera-Lavers, A., Mahoney, T. J., Hammersley, P. L., Garzón, \& F. González-Fernández, C. 2007, AJ 133, 154

Maciel, W. J., Costa, R. D. D., \& Escudero, A. 2007, IAU Symp. 245 this volume

Mahoney, T. 2007, IAU Symp. 245 this volume

Minniti, D. \& Zoccali, M. 2007, IAU Symp. 245 this volume

Ortolani, S., Renzini, A., Gilmozzi, R., Marconi, G., Barbuy, B., Bica, E., \& Rich, R. M., 1995, Nature 377, 701

Peletier, R. 2007, IAU Symp. 245 this volume

Prugniel, Ph., Maubon, G., \& Simien, F. 2001, A\&A 366, 68

Rich, R. M. 1988, AJ 95, 828

Rich, R. M. \& Origlia, L. 2005, ApJ 634, 1293

Rich, R. M., Howard, C., Reitzel, D. B., Zhao, H.-S., \& de Propis, R. 2007, IAU Symp. 245 this volume

Sil'chenko, O. 2007, IAU Symp. 245 this volume

Kobayashi, C. 2007, IAU Symp. 245 this volume

Thomas, D. 2007, IAU Symp. 245 this volume

Whitford, A. E. 1978, ApJ 226, 777

Whitford, A. E. \& Rich, R. M. 1983, ApJ 274, 723

Yi, S. K. 2007, IAU Symp. 245 this volume

Zoccali, M., Renzini, A., Ortolani, S., Greggio, L., Saviane, I., Cassisi, S., Rejkuba, M., Barbuy, B., Rich, R. M., \& Bica, E. 2003, A\&3A 399, 931

Zoccali, M., Lecureur, A., Barbuy, B., Hill, V., Renzini, A., Minniti, D., Momany, Y., Gómez, A., \& Ortolani, S. 2006, A\&A 457, L1 